\title{
PERFIL DE PACIENTES ATENDIDOS EM EMERGÊNCIA HIPERTENSIVA EM UM HOSPITAL PÚBLICO DE SALVADOR
}

\author{
PROFILE OF PATIENTS SEEN IN A HYPERTENSIVE EMERGENCY \\ IN A PUBLIC HOSPITAL IN SALVADOR
}

Lenon Cardoso Franco', Tássia Nery Faustino²

'Enfermeiro. Salvador, Bahia, Brasil. http://orcid.org/0000-0002-9971-2045. lennon.cardoso.lc@gmail.com ${ }^{2}$ Mestre em Enfermagem. Professora na Universidade do Estado da Bahia. Salvador, Bahia, Brasil.tfaustino@uneb.br

RESUMO | As emergências hipertensivas (EH) são entidades clínicas caracterizadas pela elevação aguda e significativa da pressão arterial (PA) associada a sintomas graves que evidenciam lesões de órgãos-alvo. Esse estudo objetivou traçar o perfil dos pacientes atendidos em emergência hipertensiva em um hospital público de Salvador. Trata-se de um estudo transversal descritivo. Os dados foram coletados entre $03 / 11 / 15$ e 03/02/16, através do prontuário e processados no programa SPSS versão $21 \circledR$. A estatística descritiva foi utilizada para análise dos dados. A amostra constituiu-se de 57 pacientes. A média de idade encontrada foi de 66,9 anos, com predominância do sexo feminino $(52,6 \%)$ e da cor parda $(93,0 \%)$. Aproximadamente $68,4 \%$ dos pacientes apresentavam histórico de Hipertensão Arterial Sistêmica (HAS). As EHs diagnosticadas foram o acidente vascular cerebral (AVC) isquêmico $(61,4 \%)$, seguido do AVC hemorrágico $(29,8 \%)$, da insuficiência cardíaca congestiva $(5,3 \%)$ e do infarto agudo do miocárdio (3,5\%). 28,1\% desses pacientes evoluíram com complicações. Em relação aos valores da PA encontrados na admissão, a média de pressão sistólica foi de 189,8 mmHg, enquanto que a média de pressão diastólica foi de 105,7 mmHg. Concluise que os $A V C s$, especialmente os isquêmicos, foram as $\mathrm{EH}$ mais frequentes na amostra. Faz-se necessário a efetivação de medidas de prevenção dos fatores de risco associados à HAS, assim como direcionadas ao controle dos níveis pressóricos para redução do número de atendimentos por emergência hipertensiva e demais complicações das doenças cardiovasculares.

\begin{abstract}
Hypertensive emergencies (HD) are clinical entities characterized by acute and significant elevation of blood pressure (BP) associated with severe symptoms that evidence target organ damage. This study aimed to trace the profile of patients seen in a hypertensive emergency in a public hospital in Salvador. This is a cross-sectional descriptive study. The data were collected between $03 / 11 / 15$ and $03 / 02 / 16$, through the medical record and processed in the program SPSS version $21 \circledR$. Descriptive statistics were used to analyze the data. The sample consisted of 57 patients. The mean age was 66.9 years, with a predominance of female $(52.6 \%)$ and mixed race (93.0\%). Approximately $68.4 \%$ of patients had a history of systemic arterial hypertension (SAH). The diagnosed $\mathrm{HHs}$ were ischemic stroke (61.4\%), followed by hemorrhagic stroke $(29.8 \%)$, congestive heart failure $(5.3 \%)$ and acute myocardial infarction (3.5 $\%$ ). $28.1 \%$ of these patients developed complications. In relation to BP values found on admission, the mean systolic pressure was $189.8 \mathrm{mmHg}$, while the mean diastolic pressure was $105.7 \mathrm{mmHg}$. It's concluded that the strokes, especially the ischemic ones, were the most frequent HE in the sample. It's necessary to carry out measures to prevent the risk factors associated with hypertension, as well as directed to the control of pressure levels to reduce the number of visits for hypertensive emergency and other complications of cardiovascular diseases.
\end{abstract}

Keywords: Emergencies. Hypertension / Complications. Hypertension / Diagnosis.

Palavras-chave: Emergências. Hipertensão/

Complicações. Hipertensão/Diagnóstico. 


\section{INTRODUÇÃO}

A Hipertensão Arterial Sistêmica (HAS) é uma condição clínica caracterizada por níveis elevados da pressão arterial (PA) e é definida como uma pressão arterial sistólica (PAS) maior ou igual a 140 $\mathrm{mmHg}$ ou uma pressão diastólica (PAD) maior ou igual a $90 \mathrm{mmHg}$. Entre as principais complicações da HAS descompensada encontram-se as emergências hipertensivas que são caracterizadas pela elevação aguda e grave da PA ( $\geq 180 \times 120)$ associadas a lesões de órgãos-alvo².

Pacientes em emergência hipertensiva apresentam quadros clínicos característicos e esses são mais comumente associados à Insuficiência Cardíaca Congestiva (ICC) com Edema Agudo de Pulmão, Infarto Agudo do Miocárdio (IAM) ou Angina Instável, Acidente Vascular Cerebral (AVC) isquêmico, Encefalopatia Hipertensiva, Hemorragia Intracerebral ou Subaracnóide e Dissecção de Aorta, além da Eclâmpsia no caso de gestantes ${ }^{3}$. Por conseguinte, pacientes que chegam às unidades de emergência apresentando sinais de pressão arterial muito elevada devem ser avaliados e tratados rapidamente, pois correm risco de lesões em órgãosalvo, o que poderá aumentar, significativamente, a sua morbimortalidade.

Considerando a ocorrência de lesões em órgãos-alvo, a equipe de saúde deve correr contra o tempo para que o paciente em questão não tenha maiores sequelas ou evolua com outras complicações, garantindo assim um melhor prognóstico. Ressalta-se que as emergências hipertensivas são complicações que refletem a deficiência do tratamento crônico da HAS, em que $\circ$ paciente com a doença pode ser assistido e acompanhado adequadamente pela atenção primária de saúde, evitando assim a sua ocorrência.

Associadamente, apesar da relevância da temática apresentada, verifica-se, através de pesquisa eletrônica efetuada no Portal da Biblioteca Virtual em Saúde, uma carência de publicações científicas sobre $\circ$ perfil das emergências hipertensivas diagnosticadas nas unidades de pronto-atendimento e hospitais do estado da Bahia. Face ao exposto, esse estudo objetivou traçar o perfil dos pacientes atendidos em emergência hipertensiva em um hospital público de Salvador.
Trata-se de um estudo transversal descritivo, cuja amostra foi composta por 57 pacientes em emergência hipertensiva atendidos em um hospital público estadual de grande porte do município de Salvador, Bahia. Determinou-se como critérios de inclusão: pacientes maiores de 18 anos admitidos ou que cursaram com elevação significativa da pressão arterial $(\geq 180 \times 120 \mathrm{mmHg})$ associada à lesões de orgãos-alvo. Foram excluídos da pesquisa os pacientes com registro incompleto das variáveis pesquisadas.

Os dados foram coletados entre 03 de novembro de 2015 e 03 de fevereiro de 2016, através do prontuário e foram transcritos no instrumento elaborado pelo pesquisador. Foi realizada a busca ativa dos pacientes no setor de emergência, nas unidades de terapia intensiva (UTIs) e na Unidade de AVC dessa organização hospitalar. Os pacientes que fizeram parte desse estudo foram selecionados através do censo diário das unidades, sendo então procedida a análise dos prontuários para verificação do diagnóstico e das condições clínicas na admissão.

Os dados referentes às variáveis pesquisadas, que não foram encontrados nos prontuários, foram então fornecidos pelos familiares dos participantes da pesquisa. Ressalta-se que durante o período da coleta de dados, 80 pacientes foram selecionados para $\circ$ estudo, contudo apenas 57 destes apresentavam $\circ$ registro completo das variáveis pesquisadas, constituindo-se então a amostra final.

Os dados coletados foram digitados no programa computacional Microsoft $₫$ Excel e analisados pelo programa Statitical Package for the Social Science (SPSS) versão $21 \circledR$. As variáveis de interesse desse estudo constituem-se em: variáveissociodemográficas, variáveis relativas à hipertensão arterial e variáveis relativas às emergências hipertensivas identificadas.

A pesquisa se embasou na estatística descritiva para análise dos dados, sendo que as variáveis contínuas foram expressas como média e desviopadrão (DP) e as variáveis categóricas expressas em valores absolutos e percentuais. A análise dos 
resultados ocorreu mediante comparações com produções selecionadas no Portal da Biblioteca Virtual em Saúde e no PubMed.

Esse estudo foi aprovado pelo Comitê de Ética em Pesquisa da Universidade do Estado da Bahia, CAEE $n^{\circ}$ 43783815.4.0000.0057. Todos os participantes da pesquisa ou os responsáveis pelo internamento do paciente assinaram o Termo de Consentimento Livre e Esclarecido. Durante todo o estudo foram seguidas as recomendações contidas na Resolução 466/2012 $2^{4}$.

\section{RESULTADOS}

A partir do traçado do perfil sociodemográfico da amostra, exposto na tabela 1, verificou-se que a média de idade encontrada foi de 66,9 anos, variando de 28 a 92 anos, com predominância de indivíduos do sexo feminino $(52,6 \%)$, da cor parda $(93,0 \%)$ e solteiros $(56,1 \%)$. Considerando o nível de escolaridade do presente estudo, foi observado que cerca de $63,0 \%$ da amostra tinha apenas o ensino fundamental, completo ou incompleto.

Tabela 1. Perfil sociodemográfico de pacientes em emergência hipertensiva atendidos em um hospital público de Salvador entre outubro de 2015 a janeiro de 2016.

\begin{tabular}{lcc}
\hline \multicolumn{1}{c}{ Variáveis } & $\mathbf{N}$ & $\%$ \\
\hline Idade (Média \pm DP) & $66,9 \pm 14,4$ & \\
Sexo & & \\
Masculino & 27 & 47,4 \\
Feminino & 30 & 52,6 \\
Etnia & & \\
Branco & 1 & 1,8 \\
Negro & 3 & 5,3 \\
Pardo & 53 & 93,0 \\
Estado civil & & \\
Solteiro & 32 & 56,1 \\
Casado & 14 & 24,6 \\
Divorciado & 4 & 7,0 \\
Viúvo & 5 & 8,8 \\
União estável & 2 & 3,5 \\
Escolaridade & & \\
Analfabeto & 9 & 15,8 \\
Ensino fundamental completo & 19 & 33,3 \\
Ensino fundamental incompleto & 17 & 29,8 \\
Ensino médio completo & 7 & 12,3 \\
Ensino médio incompleto & 5 & 8,8 \\
\hline DP: desvio-padrão. & &
\end{tabular}

Considerando o perfil clínico em relação ao diagnóstico de HAS (Tabela 2), 68,4\% dos pacientes apresentavam histórico da doença e $69,2 \%$ desses indivíduos haviam sido diagnosticados em um período compreendido entre 11 e 20 anos. Entre os pacientes declarados hipertensos, apenas 46,1\% faziam uso de fármacos para o controle da pressão arterial. Quanto às medicações em uso, o medicamento Losartana foi o mais utilizado $(44,4 \%)$ e dentre os pacientes que utilizavam anti-hipertensivos, $94,4 \%$ faziam uso regular dessas substâncias. 


\begin{tabular}{lcc}
\hline \multicolumn{1}{c}{ Variáveis } & N & $\%$ \\
\hline Histórico de HAS & 39 & 68,4 \\
Sim & 7 & 12,3 \\
Não & 10 & 17,5 \\
Não sabe & 1 & 1,8 \\
Não informado no prontuário & & \\
Anos do diagnóstico de HAS & 2 & 5,1 \\
5-1Oanos & 27 & 69,2 \\
$11-20$ anos & 9 & 23,1 \\
Acima de 21 anos & 1 & 2,6 \\
Não sabe informar & & \\
Medicações em uso para controle pressórico & 8 & 44,4 \\
Losartana & 4 & 22,2 \\
Captopril & 3 & 16,7 \\
Hidroclorotiazida & 3 & 16,7 \\
Terapia anti-hipertensiva combinada & & \\
Uso regular das medicações & 17 & 94,4 \\
Sim & 1 & 5,6 \\
Não & &
\end{tabular}

Dentre o perfil das emergências hipertensivas diagnosticadas na população do estudo (Tabela 3), o acidente vascular cerebral isquêmico correspondeu a $61,4 \%$ dos casos, seguido do acidente vascular cerebral hemorrágico $(29,8 \%)$, da insuficiência cardíaca congestiva $(5,3 \%)$ e do infarto agudo do miocárdio $(3,5 \%)$. Ressalta-se que $28,1 \%$ desses pacientes evoluíram com complicações, sendo a hipertensão intracraniana (HIC) a mais encontrada (15,8\%) nos paciente com AVC. Verificou-se também que $59,6 \%$ da amostra foi admitida em emergência hipertensiva e $40,4 \%$ cursou com o quadro durante o internamento.

Tabela 3. Perfil das emergências hipertensivas diagnosticadas em um hospital público de Salvador entre outubro de 2015 a janeiro de 2016.

\begin{tabular}{|c|c|c|}
\hline Variáveis & $\mathbf{N}$ & $\%$ \\
\hline \multicolumn{3}{|c|}{ Espectro da emergência hipertensiva } \\
\hline Infarto agudo do miocárdio & 2 & 3,5 \\
\hline AVC isquêmico & 35 & 61,4 \\
\hline AVC hemorrágico & 17 & 29,8 \\
\hline \multirow{2}{*}{\multicolumn{3}{|c|}{ Níveis pressóricos na admissão }} \\
\hline & & \\
\hline PAS (Média \pm DP) & $189,8 \pm 21,6$ & \\
\hline PAD (Média \pm DP) & $105,7 \pm 19,3$ & \\
\hline \multicolumn{3}{|c|}{ Emergência hipertensiva na admissão } \\
\hline Sim & 34 & 59,6 \\
\hline Não & 23 & 40,4 \\
\hline \multicolumn{3}{|l|}{ Complicações apresentadas } \\
\hline Hidrocefalia & 2 & 3,5 \\
\hline $\mathrm{HIC}$ & 9 & 15,8 \\
\hline Choque circulatório & 1 & 1,8 \\
\hline Crise convulsiva & 1 & 1,8 \\
\hline IRA & 1 & 1,8 \\
\hline IRpA & 2 & 3,5 \\
\hline Não apresentou & 41 & 71,9 \\
\hline
\end{tabular}

AVC: acidente vascular cerebral; PAS: pressão arterial sistólica; PAD: pressão arterial diastólica; FR: frequência respiratória; DP: desvio-padrão; HIC: hipertensão intracraniana; IRA: insuficiência renal aguda; IRpA: insuficiência respiratória aguda. 


\section{DISCUSSÃO}

No estudo em tela, cerca de $70,0 \%$ da amostra era composta por idosos, refletindo na média de idade encontrada. Quanto maior a idade, maior o risco de desenvolver HAS, já que as próprias alterações do envelhecimento tornam $\circ$ indivíduo mais propenso ao desenvolvimento dessa e de outras doenças e se não houver o cuidado adequado o risco de desenvolver uma lesão de órgão-alvo aumenta exponencialmente 5 .

Os resultados encontrados nessa pesquisa estão em consonância com os encontrados por estudos que observaram que a probabilidade de desenvolver uma emergência hipertensiva aumenta significativamente com $\circ$ avançar da idade e que indivíduos com idade entre 60 e 80 anos representam o grupo de risco ${ }^{6}$.

Quanto ao sexo, o presente estudo teve uma discreta predominância de participantes do sexo feminino. Sabe-se que a prevalência global de HAS entre homens e mulheres é semelhante, embora seja mais elevada nos homens até os 50 anos, invertendo-se a partir da $5^{a}$ década de vida ${ }^{7}$. Em relação à etnia, a maioria da amostra desse estudo autorreferiu ser da cor não branca e sabe-se que HAS é duas vezes mais prevalente nessa população ${ }^{7}$.

Considerando o nível de escolaridade, observouse que cerca de $63 \%$ da amostra tinha apenas o ensino fundamental completo ou incompleto. Estudos informam que, geralmente, pessoas com baixa instrução apresentam um maior número de doenças crônicas, face à expressiva influência do nível de escolaridade na qualidade e condições de vida dos indivíduos ${ }^{8}$.

No que tange ao uso de medicações, menos da metade dos pacientes declarados hipertensos fazia uso de fármacos revelando uma baixa adesão ao tratamento da HAS. Nota-se então que o tratamento da HAS engloba o reconhecimento de fatores sociodemográficos e econômicos, atitudes, crenças, percepções, expectativas e hábitos do paciente para 0 alcance das metas terapêuticas'. Verificouse também que cerca de $18 \%$ dos pacientes admitidos em emergência hipertensiva não sabiam que eram hipertensos. Com isso, faz-se necessário uma maior disponibilidade do número de consultas na atenção básica, objetivando um maior número de atendimentos e colaborando para identificação precoce e acompanhamento regular dos pacientes hipertensos.

Com relação à terapia anti-hipertensiva, a monoterapia era a mais utilizada pelos pacientes do estudo. O captopril e a hidroclorotiazida fazem parte das classes terapêuticas amplamente utilizadas para o tratamento da HAS ${ }^{10}$. Entretanto, a população afrodescendente não responde bem ao tratamento anti-hipertensivo com fármacos betabloqueadores, aqueles que atuam no sistema renina angiotensina $e$ os bloqueadores dos receptores tipo 1 da angiotensina $\mathrm{II}^{11}$. Outro dado que chama atenção é que quase a totalidade dos hipertensos que utilizavam medicação, referiu o uso regular das mesmas, inferindo a necessidade de acompanhamento regular dessa população. Nesse sentido, nota-se a importância do paciente hipertenso ser acompanhado mensalmente para verificar-se a necessidade de alterar a dose ou modificar as medicações em uso, de acordo com os níveis pressóricos e da sintomatologia referida pelo mesmo ${ }^{12}$.

Em relação aos valores de PA dos participantes da pesquisa, os da PAS estavam próximos dos apresentados pela literatura para a ocorrência de emergência hipertensiva ${ }^{2}$, contudo os valores da PAD estavam abaixo dos comumente encontrados. Sabe-se que 0 tratamento do paciente em emergência hipertensiva geralmente se inicia quando a PA encontra-se acima de $180 \times 120$ $\mathrm{mmHg}$, mas este nível de pressão não é obrigatório, visto que alguns pacientes podem apresenta-la com valores pressóricos menores ${ }^{13}$. Com isso, reitera-se a importância do profissional de saúde em atentar-se não apenas aos valores de PA apresentados, mas associá-los sempre aos sinais e sintomas referidos pelos pacientes e, por conseguinte, à ocorrência de emergências hipertensivas.

O hospital em que foi realizado este estudo é referência estadual no atendimento às doenças cerebrovasculares, justificando a maior parte da amostra que apresentava esse espectro de emergência hipertensiva. Quanto às afecções cardiovasculares identificadas, a amostra foi bem 
menor, correspondendo a 3 casos de ICC e 2 casos de IAM.

No estudo observou-se que cerca de $41 \%$ evoluiu com emergência hipertensiva durante o internamento. Esta é uma particularidade dos pacientes diagnosticados com AVC do tipo isquêmico. A HAS é comum nesse perfil de paciente, pois relaciona-se à compensação fisiológica devido a isquemia cerebral e aumento reativo na PAM secundário à elevação progressiva da pressão intracraniana ${ }^{13,14}$. Contudo, como estudos têm revelado piora neurológica associada à redução da $\mathrm{PA}$, especialmente no primeiro dia de AVC isquêmico, toleram-se níveis pressóricos de PAS até $220 \mathrm{mmHg}$ e PAD até $120 \mathrm{mmHg}$ nas primeiras 24 horas do evento ${ }^{14}$.

Por conseguinte, a HAS só é tratada no AVC isquêmico quando os níveis pressóricos ultrapassarem os valores determinados e persistirem por mais de 24 horas; quando o paciente cursar com outras emergências hipertensivas associadas; ou quando o paciente tem indicação de trombólise, nesse caso a PA deve ser mantida abaixo de $185 \times 110 \mathrm{mmHg}$ antes do procedimento ${ }^{14}$.

A maior parte da amostra do presente estudo não evoluiu com complicações. Reitera-se com esse resultado a importância da identificação e tratamento precoce das emergências hipertensivas, buscando evitar desfechos clínicos negativos. A HIC foi a complicação mais frequente nos pacientes com AVC hemorrágico. Esta complicação nesse espectro do AVC é decorrente do volume de sangue que se mistura ao volume liquórico, comprometendo a reabsorção liquórica no nível das granulações aracnóideas ou levando à obstrução no sistema ventricular, aumentando consequentemente a pressão intracraniana ${ }^{15}$.

\section{CONCLUSÃO}

Este estudo revelou que os $\mathrm{AVCs}$, especialmente os isquêmicos, constituíram as emergências hipertensivas mais frequentes em um hospital público de Salvador. Associadamente, pacientes idosos, do sexo feminino e da etnia autorreferida parda foram os mais acometidos. Quanto ao perfil clínico em relação ao diagnóstico de HAS, a maioria dos pacientes apresentava histórico da doença e fazia uso de monoterapia medicamentosa.

Sabe-se que a HAS é uma doença silenciosa e que muitos pacientes não sabem que convivem com essa comorbidade, como foi o caso de uma parcela da amostra dessa pesquisa. Com isso, faz-se necessário a efetivação de medidas de prevenção dos fatores de risco associados à HAS, assim como direcionadas ao controle dos níveis pressóricos, para redução do número de atendimentos por emergência hipertensiva e demais complicações das doenças cardiovasculares.

Dentre as limitações desse estudo destaca-se a expressiva perda de participantes devido ao registro incompleto das variáveis pesquisadas no prontuário. Adicionalmente, algumas informações foram fornecidas por familiares dos pacientes, como a estimativa do número de anos de diagnóstico da HAS, o que poderia não corresponder à realidade.

Como o lócus em que a pesquisa foi realizada é referência no atendimento a doenças cerebrovasculares e pelo fato da capital baiana contar com um hospital de referência para doenças cardiovasculares, recomenda-se a realização de estudos com amostras representativas, abrangendo os hospitais públicos gerais e especializados de grande porte de Salvador, objetivando traçar um panorama mais completo da ocorrência de emergências hipertensivas na população soteropolitana.

\section{CONFLITOS DE INTERESSES}

Nenhum conflito financeiro, legal ou político envolvendo terceiros (governo, empresas e fundações privadas, etc.) foi declarado para nenhum aspecto do trabalho submetido (incluindo mas não limitandose a subvenções e financiamentos, conselho consultivo, desenho de estudo, preparação de manuscrito, análise estatística, etc). 


\section{REFERÊNCIAS}

1. Sociedade Brasileira de Hipertensão. IV Diretrizes Brasileiras de Hipertensão Arterial. Arquivos Brasileiros de Cardiologia. 2014;82(1):7-22.

2. Rodriguez MA, Kumar SK, de Caro M. Hypertensive Crisis. Cardiology in review. 2010;18(1):102-107. doi: $10.1097 /$ CRD.0b013e3181c307b7

3. Souza JAM et al. Emergências Hipertensivas. In: KNOBEL, Elias. Condutas no paciente grave. 3 ed. São Paulo: Atheneu; 2006. p. 271-280.

4. Brasil. Ministério da Saúde. Conselho Nacional de Saúde. Comissão de Ética em Pesquisa. Resolução N 466/2012, Brasília. DF. 2012.

5. de Souza ARA, Costa A, Nakamura D, Mocheti LN, Filho PRS, Ovando LA. Um estudo sobre Hipertensão Arterial Sistêmica na cidade de Campo Grande, MS. Arquivos Brasileiros de Cardiologia. 2007;88(4):441-446. doi: $10.1590 /$ S0066-782X2007000400013

6. Salkic S, Ljuca F, Batic-Mujanovic O, Brkic S, Mesic D, Mustafic $S$. The Frequency of Hypertension Crises in the Emergency Medical Service Department in Tuzla. Medical Archives Journal. 2013;67(2):393-396. doi: 10.5455/ medarh.2013.67.393-396

7. Sociedade Brasileira de Cardiologia. VI Diretrizes Brasileiras de Hipertensão. Arquivos Brasileiros de Cardiologia. 2010;95(1):1-51. doi: 10.1590/S0066$\underline{782 \times 2010001700001}$

8. Andrade SSA, Stopa SR, Brito AS, Chueri PS, Szwarcwald $\mathrm{CL}$, Malta DC. Prevalência de hipertensão arterial autorreferida na população brasileira: análise da Pesquisa Nacional de Saúde, 2013. Epidemiologia dos Serviços de Saúde. 2015;24(2):297-304. doi: 10.5123/S167949742015000200012

9. Rodrigues MTP, Moreira TMM, de Andrade DF. Elaboração e validação de instrumento avaliador da adesão ao tratamento da hipertensão. Revista de Saúde Pública. 2014;48(2): 232-239. doi: 10.1590/S0034$\underline{8910.2014048005044}$

10. da Silva DB, de Souza TA, dos Santos CM, Jucá $M M$, Moreira TMM, Frota MA et al. Associação entre Hipertensão Arterial e Diabetes em Centro de Saúde da Família. Revista Brasileira em Promoção da Saúde. 201 1;24(1):16-23. doi:10.5020/18061230.2011.p16

11. Pereira SE, Aguiar RS, Vian VH. Análise crítica do atendimento da crise hipertensiva em Unidade de Pronto Atendimento de Itaperuma - Rio de Janeiro. Revista Brasileira de Medicina. 2010;12(6):139-144.
12. Brasil. Ministério da Saúde. Cadernos de Atenção Básica. Estratégias para o cuidado da pessoa com doença crônica, Hipertensão Arterial Sistêmica. Brasília. DF. 2013.

13. Feitosa-Filho GS, Lopes RD, Poppi NT, Guimarães HP. Emergências Hipertensivas. Revista Brasileira de Terapia Intensiva. 2008;20(3):305-312. doi: 10.1590/S0103$\underline{507 \times 2008000300014}$

14. Oliveira-Filho J, Martins SCO, Pontes-Neto OM, Longo A, Evaristo EF, de Carvalho JJF et al. Guidelines for acute ischemic stroke treatment - Part I. Arquivos de Neuropsiquiatria. 2012;70(8):621-629. doi: 10.1590/ $\underline{\text { S0004-282X2012000800012 }}$

15. Schettino G, Cardoso LF, JM Junior, Filho FT. Paciente crítico: diagnóstico e tratamento: Hospital Sírio Libanês. Barveri, SP: Manole; 2006. 